\title{
THERMODYNAMICS OF THE 1+1-DIMENSIONAL NONLINEAR SIGMA MODEL THROUGH NEXT-TO-LEADING ORDER IN $1 / N$
}

\author{
HARMEN J. WARRINGA \\ Department of Physics and Astronomy, Vrije Universiteit, \\ De Boelelaan 1081, 1081 HV Amsterdam, The Netherlands \\ E-mail: harmen@nat.vu.nl
}

\begin{abstract}
We discuss the thermodynamics of the $O(N)$ nonlinear sigma model in $1+1$ dimensions. In particular we investigate the NLO $1 / N$ correction to the $1 \mathrm{PI}$ finite temperature effective potential expressed in terms of an auxiliary field. The effective potential contains temperature-dependent divergences which cannot be renormalized properly. We argue that this problem vanishes at the minimum of the effective potential. Therefore physical quantities like the pressure are well defined and can be renormalized in a temperature-independent way. We give a general argument for the occurrence of temperature-dependent divergences outside the minimum. We present calculations of the pressure and show that $1 / N$ is a good expansion. It turns out that the pressure normalized to that at infinite temperature is $N$ independent like the flavor independence of the same quantity in QCD.
\end{abstract}

\section{Introduction}

It is interesting to study the thermodynamics of the $O(N)$ nonlinear sigma model (NLSM) in $1+1$ dimensions in a $1 / N$ expansion. It allows one to learn about renormalization at finite temperature $(T)$ in a non-perturbative approach. In general one expects that temperature does not influence the ultraviolet behavior of the theory. Hence if the theory is renormalized at $T=0$, it should also be renormalized at finite $T$. However, this statement is not true for the effective potential as a function of the vacuum expectation value (vev) of an auxiliary field as we will see. The 1+1-dimensional NLSM can also be used as a toy model to study QCD. This is because both theories are asymptotically free. Unlike in large $N_{c}$ QCD one can calculate the pressure of the NLSM through NLO in $1 / N$. By performing this calculation we were able to test the $1 / N$ expansion at finite temperature. 
The $O(N)$ symmetric NLSM is defined by the Lagrangian density

$$
\mathcal{L}=\frac{1}{2} \partial_{\mu} \phi_{i} \partial^{\mu} \phi_{i}, \quad \phi_{i}(x) \phi_{i}(x)=\frac{N}{g_{b}^{2}}, \quad i=1, \ldots, N,
$$

where $g_{b}$ is the bare coupling constant and $\phi_{i}$ are scalar fields which are constrained to live on a hypersphere. The NLSM is renormalizable because $g_{b}$ is dimensionless. It is asymptotically free and contains infrared renormalon ambiguities ${ }^{1}$. The scalar fields become massive due to the interactions. The mass is non-analytic in the coupling constant.

The effective potential of the NLSM has been studied through NLO in the $1 / N$ expansion at $T=0^{2,3,4,5,6}$. The LO contribution at finite $T$ was also investigated before ${ }^{7}$. We studied the NLO correction at finite $T^{8}$.

\section{The effective potential}

A way to implement the constraint on the $\phi$ fields is by introducing an auxiliary Lagrange multiplier field which we will denote by $\alpha$. The action then still remains quadratic in the $\phi$ fields. If one integrates over the $\phi$ fields one finds the partition function of the NLSM which is

$$
\mathcal{Z}=\int \mathcal{D} \alpha \exp \left\{-\frac{N}{2} \operatorname{Tr} \log \left[-\partial^{2}-i \alpha(x)\right]-\frac{i N}{2 g_{b}^{2}} \int_{0}^{1 / T} \mathrm{~d} \tau \int \mathrm{d} x \alpha(x)\right\} .
$$

The effective potential can be calculated from the partition function by expanding the $\alpha$ field around its vev. One can prove that the vev of the $\alpha$ field is purely imaginary. Therefore we write $\alpha=i m^{2}+\tilde{\alpha} / \sqrt{N}$. By expanding the $\operatorname{Tr} \log$ term of Eq. (2) around $i m^{2}$, one sees that $\tilde{\alpha}$ n-point vertices are suppressed with respect to the propagator in the large- $N$ limit. Hence, the effective potential can be calculated in a $1 / N$ expansion. The LO contribution stems from the classical action of the $\alpha$ field. The NLO correction can be found by performing the Gaussian integral over the quantum fluctuations $\tilde{\alpha}$. One finds $\mathcal{V}\left(m^{2}\right)=N \mathcal{V}_{\mathrm{LO}}\left(m^{2}\right)+\mathcal{V}_{\mathrm{NLO}}\left(m^{2}\right)$ where

$$
\mathcal{V}_{\mathrm{LO}}=\frac{m^{2}}{2 g_{b}^{2}}-\frac{1}{2} \mathcal{F}_{P} \log \left(P^{2}+m^{2}\right), \quad \mathcal{V}_{\mathrm{NLO}}=-\frac{1}{2} \mathcal{f}_{P} \log \Pi(P, m) .
$$

The Fourier transform of the inverse $\tilde{\alpha}$ propagator is given by

$$
\Pi(P, m)=\frac{1}{2} \mathcal{F}_{Q} \frac{1}{(P+Q)^{2}+m^{2}} \frac{1}{Q^{2}+m^{2}} .
$$

For convenience we use the following notation, $P=\left(p_{0}, p\right)$ and

$$
\int_{P} \equiv \int \frac{\mathrm{d}^{2} p}{(2 \pi)^{2}}, \quad \mathcal{f}_{P} \equiv T \sum_{p_{0}=2 \pi n T} \int \frac{\mathrm{d} p}{2 \pi}, \quad \mathcal{\psi}_{P} \equiv \mathcal{F}_{P}-\int_{P} .
$$


To deduce the required renormalization of the effective potential, we write $\mathcal{V}=N m^{2} / 2 g_{b}^{2}+N D_{\mathrm{LO}}+N F_{\mathrm{LO}}+D_{\mathrm{NLO}}+F_{\mathrm{NLO}}$, where $F_{\mathrm{LO}}$ and $F_{\mathrm{NLO}}$ are finite quantities which have to be calculated numerically. The terms $D_{\mathrm{LO}}$ and $D_{\mathrm{NLO}}$ are divergent and will be calculated analytically. The theory is infrared finite, so the divergences will arise from the high-momentum modes. To regulate the ultraviolet divergences, we introduce a momentum cutoff $\Lambda$. The quantity $\oint_{P} \log [f(P, m)]$ is finite due to Bose-Einstein suppression. Hence the divergences of the effective potential stem from $\int_{P} \log \left[\lim _{P \gg m, T} f(P, m)\right]$. In this way one finds $D_{\mathrm{LO}}=-m^{2} \log \left(\Lambda^{2} / m^{2}\right) / 8 \pi$. It turns out ${ }^{8}$ that $\Pi(P, m)$ has an explicit $T$-dependence in the large-momentum limit. As a result we find

$$
\begin{aligned}
D_{\mathrm{NLO}}=-\frac{1}{8 \pi}\left[\Lambda^{2} \log \log \left(\frac{\Lambda^{2}}{\bar{m}^{2}}\right)\right. & \left.-\bar{m}^{2} \operatorname{li}\left(\frac{\Lambda^{2}}{\bar{m}^{2}}\right)\right] \\
+ & \frac{m^{2}}{4 \pi}\left[\log \left(\frac{\Lambda^{2}}{m^{2}}\right)-\log \log \left(\frac{\Lambda^{2}}{\bar{m}^{2}}\right)\right],
\end{aligned}
$$

which is $T$-dependent because $\bar{m}^{2}=m^{2} \exp \left[-J_{1}(\beta m)\right]$. Here $J_{1}(x)=$ $4 \int_{0}^{\infty} \mathrm{d} t n\left(\omega_{t}\right) / \omega_{t}$ with $\omega_{t}=\sqrt{t^{2}+x^{2}}$ and $n(x)=[\exp (x)-1]^{-1}$. Hence the quadratic and the li-divergence have an explicit $T$-dependence. The logarithmic integral is defined by $\operatorname{li}(x)=\mathcal{P} \int_{0}^{x} \mathrm{~d} t 1 / \log t$.

One possibility to remove the divergences of $\mathcal{V}$ is by subtracting infinite constants independent of $T$ and $m^{2}$. This is allowed because it does not change the shape of $\mathcal{V}$ and hence not the physics. Clearly this cannot remove the quadratic divergences, because $\Lambda^{2}\left[\log \log \left(\Lambda^{2} / \bar{m}^{2}\right)-\log \log \left(\Lambda^{2} / \mu^{2}\right)\right]$ is still divergent. The second possibility is to absorb $T$-independent divergences into $g_{b}$. This does not solve the problem of the li-divergence, since it is proportional to $\bar{m}^{2}$, whereas $1 / g_{b}^{2}$ is proportional to $m^{2}$ in $\mathcal{V}$. The $m^{2} \log \log \left(\Lambda^{2} / \bar{m}^{2}\right)$ divergence can however be renormalized in this way. The two problematic divergences together are called 5 "perturbative tail" and do not arise in dimensional regularization. One can remove these divergences by subtracting the perturbative tail. This however gives rise to a $T$-dependent ambiguity due to a thermal infrared renormalon ${ }^{8}$.

We therefore conclude that we cannot renormalize $\mathcal{V}$ in a $T$-independent way. However, in the minimum of $\mathcal{V}, \bar{m}^{2}=\Lambda^{2} \exp \left(-4 \pi / g_{b}^{2}\right)+\mathcal{O}(1 / N)^{8}$. Furthermore one can show ${ }^{2}$ that one only needs the LO expression for $m^{2}$ and $\bar{m}^{2}$ to calculate $\mathcal{V}$ in the minimum. Hence the divergences become $T$-independent in the minimum and they can be removed by subtracting a vacuum term. Therefore quantities which are to be calculated in the minimum are well defined. This holds for example for the pressure $\mathcal{P}$. A 
summary of the different ways to deal with $\mathcal{V}$ is shown in Table 1.

Table 1. Different ways to deal with the effective potential and their problems. The subscript min indicates the minimum of the effective potential.

\begin{tabular}{c|l} 
quantity & problem \\
\hline \hline $\mathcal{V}\left(m^{2}\right)$ & $T$-dependent divergences \\
$\mathcal{V}\left(m^{2}\right)-\mathcal{V}\left(m^{2}\right)_{T=0}$ & changes shape $\mathcal{V}$ and $T$-dependent divergences \\
$\mathcal{V}\left(m^{2}\right)-\mathcal{V}_{\text {min }, T=0}$ & $T$-dependent divergences \\
$\mathcal{V}\left(m^{2}\right)-$ pert. tail & $T$-dependent infrared renormalon ambiguity \\
$\mathcal{P}=\mathcal{V}_{\text {min }}-\mathcal{V}_{\text {min }, T=0}$ & no problem
\end{tabular}

\section{Temperature-dependent divergences}

In this section we will argue in a more general way why it is possible to have $T$-dependent divergences outside the minimum of the effective potential. These $T$-dependent divergences are not specific for the NLSM but also occur in the linear sigma model in $d=3+1^{9}$. We add a space-time independent source $J$ for $\alpha$ and define the action in the presence of $J$ as

$$
S[J]=\frac{N}{2} \operatorname{Tr} \log \left[-\partial^{2}-i \alpha(x)\right]+i\left(\frac{N}{2 g_{b}^{2}}+J\right) \int_{0}^{1 / T} \mathrm{~d} \tau \int \mathrm{d} x \alpha(x) .
$$

The quantity $Z[J]_{T}$ is the partition function of the theory with action $S[J]$ at temperature $T$. If we assume that $\log Z[0]_{T}$ does not contain $T$-dependent divergences this also holds for $\log Z[J]_{T}$ as long as $J$ is $T$ independent. This is because any $J$ can be incorporated in $1 / g_{b}^{2}$ by a redefinition. The effective potential in the minimum is equal to $\log Z[0]_{T}$. Using this one can deduce from Eq. (6) that the divergences of $\log Z[0]_{T}$ depend on $1 / g_{b}^{2}$. Therefore varying $J$ will change the divergences of $\log Z[J]_{T}$.

The effective potential is given by $\mathcal{V}\left(m^{2}\right)=\log Z[J]_{T} / \beta V-J m^{2}$, where $J$ is now the current that gives the $\alpha$ field the vev $i m^{2}$ at a certain $T$. If one varies $T$, the implicitly defined $J$ has to change in order to keep the same vev $\mathrm{im}^{2}$. Therefore $J$ depends on $T$ and as we argued before varying $J$ changes the divergences. Outside the minimum of the effective potential $J$ is nonzero. This implies that the effective potential outside the minimum can contain $T$-dependent divergences.

\section{Pressure}

For the arbitrary choice of $g^{2}(\mu=500$ a.u. $)=10$, we calculated the pressure $\mathcal{P}$ as function of $T$ for different values of $N^{8}$. The results are depicted in Fig. 1. With a.u. we indicate that all results are in arbitrary units. 

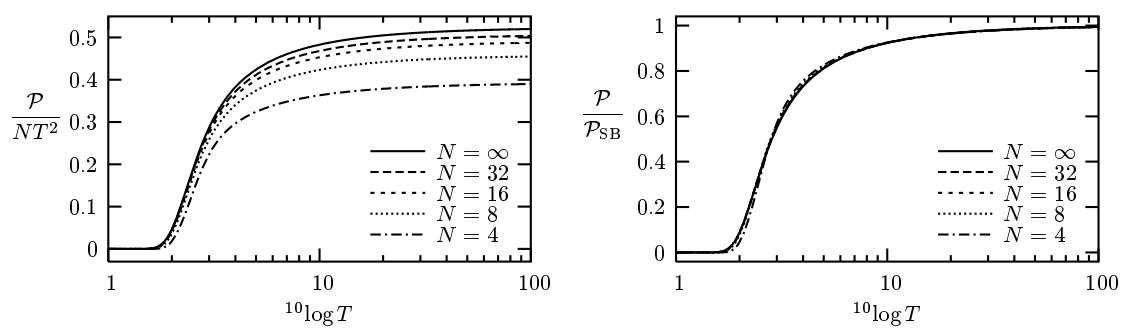

Figure 1. Pressure $\mathcal{P}$ normalized to $N T^{2}$ (left) and normalized to $\mathcal{P}(T=\infty)$ (right), as a function of $T$, for different values of $N$ with $g^{2}(\mu=500)=10$.

One can see from Fig. 1 that the NLSM shows a kind of cross-over transition. This is because at low $T$ the theory is strongly interacting and hence the degrees of freedom are effectively frozen. Due to asymptotic freedom, at high $T$ the pressure is approaching that of a free gas of $N-1$ bosonic degrees of freedom, i.e., $\mathcal{P}=\mathcal{P}_{\mathrm{SB}}=(N-1) \pi T^{2} / 6$. Figure 1 also shows that the $1 / N$ expansion is a good expansion for the NLSM. This is because $1 / N$ corrections are really of order $1 / N$. The pressure divided by the pressure at $T=\infty$ is almost independent of $N$. Similar behavior is shown by QCD lattice calculations with and without dynamical quarks in $d=3+1^{10}$, where the same quantity is flavor independent.

\section{Acknowledgments}

This work has been carried out in collaboration with Jens O. Andersen and Daniël Boer. The author would like to thank the organizers of the strong and electroweak matter conference for the stimulating meeting and the opportunity to present this work.

\section{References}

1. F. David, Nucl. Phys. B 209, 433 (1982).

2. R. G. Root, Phys. Rev. D 10, 3322 (1974).

3. V. A. Novikov, M. A. Shifman, A. I. Vainshtein and V. I. Zakharov, Phys. Rept. 116, 103 (1984).

4. C. Rim and W. I. Weisberger, Phys. Rev. Lett. 53, 965 (1984).

5. P. Biscari, M. Campostrini and P. Rossi, Phys. Lett. B 242, 225 (1990).

6. H. Flyvbjerg, Phys. Lett. B 245, 533 (1990).

7. M. Dine and W. Fischler, Phys. Lett. B 105, 207 (1981).

8. J. O. Andersen, D. Boer and H. J. Warringa, Phys. Rev. D 69076006 (2004).

9. J. O. Andersen, D. Boer and H. J. Warringa, arXiv:hep-ph/0408033.

10. F. Karsch, E. Laermann and A. Peikert, Phys. Lett. B 478, 447 (2000). 